\title{
Equação de volume para apoio ao manejo comunitário de empreendimento florestal em Anapu, Pará
}

\author{
Wallacy Ferreira Barreto ${ }^{1}$, Fábio Miranda Leão², Marlon Costa de Menezes², Deivison Venicio Souza ${ }^{2}$ \\ ${ }^{1}$ Instituto Floresta Tropical - IFT, Avenida Brigadeiro Eduardo Gomes, São Sebastião, CEP 68.372-040, Altamira, PA, Brasil \\ ${ }^{2}$ Universidade Federal do Pará, Campus de Altamira, Rua Coronel José Porfirio, 2515, São Sebastião, CEP 68.372-040, Altamira, PA, Brasil
}

*Autor correspondente:

fabioleao@ufpa.br

Termos para indexação:

Assentamento

floresta Amazônica

modelo Husch

Index terms:

Settlementt

Amazonia forest

Husch model

Histórico do artigo:

Recebido em 02/06/2014

Aprovado em 18/12/2014

Publicado em 31/12/2014

doi: 10.4336/2014.pfb.34.80.721
Resumo - O manejo florestal comunitário em áreas de assentamento, no Pará, necessita de ferramentas que forneçam informações confiáveis sobre o potencial madeireiro da área, como equações de volume. Foram ajustados cinco modelos volumétricos de simples entrada e cinco de dupla entrada para estimar o volume de madeira em pé no Projeto de Desenvolvimento Sustentável Virola-Jatobá em Anapu, Pará. Foram cubados, pelo método de Smalian, 172 indivíduos com diâmetro tomado a 1,30 m do solo de 60 a $120 \mathrm{~cm}$ e altura comercial de 5,24 a $35 \mathrm{~m}$, de 25 espécies de interesse comercial da comunidade. Utilizaram-se os critérios estatísticos coeficiente de determinação ajustado ( $\left.\mathrm{R}^{2} \mathrm{aj}\right)$, erro padrão da estimativa (Sxyr), coeficiente de variação $(\mathrm{CV})$, desvio médio percentual (DMP) e a análise gráfica de resíduos para selecionar o melhor modelo de simples e de dupla entrada. O teste do qui-quadrado foi utilizado para validar os melhores modelos. O modelo de Husch foi confirmado para estimar o volume de madeira em pé na área de estudo, pela equação $\operatorname{logv}=0,7974+4,4294 \operatorname{logd}$. Essa equação não utiliza a variável altura comercial, por isso pode gerar menos custos na atividade de inventário e evitar erros que provém da estimativa dessa variável.

\section{Volume equation as basis of community forest management in Anapu, Pará State, Brazil}

\begin{abstract}
The community forest management in settlement areas, in Pará, needs tools that provide reliable information about wood potential in the area, as volume equations. Five volumetric models of simple entrance and five of double entrance were adjusted to estimate the wood volume of standing trees in the Project of sustainable development Virola-Jatobá in Anapu, Pará State, Brazil. The trees were cubed using Smalian method. The diameter at $1.3 \mathrm{~m}$ above soil level was measured in 172 individuals from 25 species with commercial interest by the community. The diameters ranged from 60 to 120 $\mathrm{cm}$ and commercial height varied from $5.24 \mathrm{~m}$ to $35 \mathrm{~m}$. The statistic criteria used to select the best volume model of simple or double entrance was adjusted coefficient of determination ( $\left.\mathrm{R}^{2} \mathrm{aj}\right)$, standard error of estimate (Sxyr), coefficient of variation (CV), average deviation percentage (ADP) and graphic residual analysis. Chi-square test was used to validate the best models. Husch model presented the best estimative of wood volume in standing trees in the studied area, through the equation $\operatorname{logv}=0.7974$ $+4.4294 \log$. This equation does not use the commercial height variable, so it field inventory cost less and avoid mistakes from the estimation of this variable.
\end{abstract}




\section{Introdução}

O Projeto de Desenvolvimento Sustentável (PDS) III e IV, denominado Virola-Jatobá, criado no ano de 2002 em Anapu, Pará, atende a necessidade de maior proteção dos recursos naturais e de trabalhadores rurais que migravam para aquele território, reivindicada por diversas organizações sociais, tendo em vista a ocorrência de conflitos fundiários de ampla repercussão e intensa exploração ilegal da floresta praticados na região (Pará, 2004).

Com o intuito de explorar os recursos florestais de forma racional e frear o desmatamento ilegal, decidiu-se implantar o manejo florestal comunitário no PDS VirolaJatobá, para gerar desenvolvimento socioambiental e econômico, pautado na gestão participativa, na diversificação das atividades econômicas e na capacitação dos beneficiários, devendo ser compatibilizada com a rotina produtiva e as atividades existentes, possibilitando a construção coletiva e a promoção de projetos de longo prazo (Incra, 2010).

O êxito do manejo florestal comunitário depende da execução de políticas públicas coerentes com as necessidades dos comunitários e da utilização de ferramentas que propiciem informações confiáveis sobre o potencial florestal, como a volumetria de madeira existente na área (Barros \& Silva Júnior, 2009). Sendo assim, as equações de volume são exigidas na Amazônia brasileira para o cálculo de volume em pé de madeira em áreas de manejo florestal com mais de uma unidade de produção anual (Brasil, 2009). Elas têm parâmetros determinados por regressão e são imprescindíveis na tomada de decisão do manejo florestal (Silva Júnior, 2009; Santos et al., 2012).

Equações volumétricas têm sido ajustadas na Amazônia brasileira (Fernandes, et al.,1983; Figueiredo Filho, 1983; Silva \& Carvalho, 1984; Silva \& Araújo, 1984; Silva et al., 1984; Rolim et al., 2006; Silva, 2007a; Barros \& Silva Júnior, 2009; Colpini et al., 2009; Thaines et al., 2010). Contudo, modelos volumétricos específicos para áreas de manejo florestal comunitário são escassos.

Produzir equações específicas para cada local a ser manejado é importante, pois condições distintas de clima e solo são determinantes na relação entre as variáveis diâmetro, altura e volume das árvores,. Logo, modelos baseados em um povoamento ou região de condições de clima e solo uniformes, propiciam estimativas mais precisas dessas variáveis (Loetsch et al., 1973 ; Valente et al., 2011; Santos et al., 2012). Por esse motivo, este trabalho objetivou ajustar um modelo volumétrico preciso, para espécies de interesse comercial, em um empreendimento florestal comunitário no Projeto de Desenvolvimento Sustentável Virola-jatobá em Anapu, Pará.

\section{Material e métodos}

O PDS Virola-jatobá $\left(03^{\circ} 10^{\prime} 06,4^{\prime \prime} \mathrm{S}\right.$ e $\left.51^{\circ} 17^{\prime} 55,5^{\prime \prime} \mathrm{O}\right)$ possui área de 41.640 ha, no Município de Anapu, Pará). O relevo é caracterizado por planícies e serras acentuadas, com predominância de Latossolo vermelho e Latossolo amarelo). O clima da região, segundo a classificação de Köppen, é do tipo Am com precipitações anuais entre $1.500 \mathrm{~mm}$ a $2.500 \mathrm{~mm}$ e temperatura média maior que $27^{\circ} \mathrm{C}$ e menor que $29,2^{\circ} \mathrm{C}$ (Pará, 2004). A vegetação é caracterizada como Floresta Ombrófila Densa.

Calculou-se com o auxílio de planilha eletrônica o volume real de madeira de 172 indivíduos, pelo método de Smalian segundo Machado \& Figueiredo Filho (2009), de espécies de interesse comercial, com diâmetro tomado a $1,30 \mathrm{~m}$ do solo (DAP) variando de $60 \mathrm{~cm}$ a $120 \mathrm{~cm}$ e altura comercial variando de $5,24 \mathrm{~m}$ a $35 \mathrm{~m}$.

Loetsch et al. (1973) consideram que para a construção de uma tabela de volume local, se faz necessária a cubagem de 50 a 100 árvores. No entanto, segundo o teorema do limite central, quanto maior a amostragem, menor é o erro padrão e mais próximo da normalidade o conjunto de amostras se encontrará independente de sua distribuição inicial, ou seja, quanto maior o número de amostras, mais próximo da realidade o conjunto de dados estará.

Para o ajuste dos modelos volumétricos na comunidade do PDS Virola-jatobá foram utilizados 132 indivíduos de 25 espécies de interesse comercial devidamente identificadas com auxílio do identificador botânico, registrando-se o nome vulgar regional dos indivíduos, que posteriormente foram classificadas em nível de espécie, segundo sensu Angiosperm Phylogeny Group II (APG II, 2003; Tabela 1) com DAP que variou de 60 $\mathrm{cm}$ a $120 \mathrm{~cm}$ e altura comercial de $10,9 \mathrm{~m}$ a $35 \mathrm{~m}$ (Tabela 2). Foram testados cinco modelos de simples entrada e cinco de dupla entrada dos 17 sugeridos por Loetsch et al. (1973), já utilizados no ajuste de equações volumétricas na Amazônia brasileira, também com auxilio de planilha eletrônica (Barros \& Silva Júnior, 2009; Thaines et al., 2010) (Tabela 3). 
Tabela 1. Número de indivíduos (N) por espécie utilizada para o ajuste dos modelos volumétricos no PDS Virola-jatobá em Anapu, Pará.

\begin{tabular}{|c|c|c|}
\hline Nome científico & Nome popular & $\mathbf{N}$ \\
\hline Astronium Le-cointei Ducke & Muiracatiara & 9 \\
\hline Bowdichia nitida Spruce. & Sucupira preta & 1 \\
\hline Buchenavia capitata (Vahl) Eichler & Tanibuca & 1 \\
\hline Carapa guianensis Aubl. & Andiroba & 4 \\
\hline Chrysophylum sp. & Guajará bolacha & 10 \\
\hline Cordia goeldiana Huber & Freijó cinza & 1 \\
\hline Couratari guianensis Aubl. & Tauari & 14 \\
\hline Dinizia excelsa Ducke & Angelim vermelho & 4 \\
\hline Dipteryx odorata (Aubl.) Willd & Cumaru & 2 \\
\hline Enterolobium schomburgkii (Benth) Benth & Orelha de macaco & 1 \\
\hline Goupia glabra Aubl. & Cupiuba & 2 \\
\hline Hymenaea courbaril $\mathrm{L}$. & Jatobá & 9 \\
\hline Hymenolobium petraeum Ducke & Angelim pedra & 4 \\
\hline Lecythis paraensis Huber Ex. Ducke & Sapucaia & 2 \\
\hline Licaria reitzkleiniana Vattimo & Louro pimenta & 1 \\
\hline Manilkara bidentata (A.DC.) A.Chev. & Maparajuba & 7 \\
\hline Manilkara huberi (Ducke) A.Chev. & Maçaranduba & 21 \\
\hline Ocotea baturitensis Vattimo & Louro preto & 1 \\
\hline Ocotea sp. & Louro itauba & 4 \\
\hline Peltogyne paniculata Benth. & Escorrega macaco & 1 \\
\hline Piptadenia suaveolens Miq. & Timborana & 9 \\
\hline Protium tenuifolium (Engl.) Engl. & Breu vermelho & 1 \\
\hline Screrolobium paraense Huber & Tachi branco & 7 \\
\hline Tachigali paniculata Aubl. & Tachi preto & 13 \\
\hline Vatairea sericea (Ducke) Ducke & Angelim amargoso & 3 \\
\hline Total & & 132 \\
\hline
\end{tabular}

Tabela 2. Número de indivíduos por classes de diâmetro e altura utilizados para o ajuste dos modelos volumétricos no PDS Virola-jatobá em Anapu, Pará.

\begin{tabular}{|c|c|c|c|c|c|c|c|c|}
\hline \multirow{2}{*}{$\begin{array}{l}\text { Classe de } \\
\text { DAP }\end{array}$} & \multirow{2}{*}{$\begin{array}{c}\text { Intervalo de } \\
\text { DAP (cm) }\end{array}$} & \multicolumn{6}{|c|}{ Classe de altura (m) } & \multirow{2}{*}{ Tota } \\
\hline & & $10 \mid-15$ & $15 \mid-20$ & $20 \mid-25$ & $25 \mid-30$ & $30 \mid-35$ & $\geq 35$ & \\
\hline 1 & $60-70$ & 4 & 6 & 5 & 1 & 1 & 0 & 17 \\
\hline 2 & $70-80$ & 4 & 10 & 7 & 1 & 1 & 0 & 23 \\
\hline 3 & $80-90$ & 6 & 10 & 4 & 5 & 0 & 0 & 25 \\
\hline 4 & $90-100$ & 0 & 6 & 9 & 5 & 1 & 0 & 21 \\
\hline 5 & $100-120$ & 3 & 2 & 11 & 2 & 5 & 0 & 23 \\
\hline 6 & $110-120$ & 0 & 6 & 5 & 9 & 2 & 1 & 23 \\
\hline \multicolumn{2}{|c|}{ Total } & 17 & 40 & 41 & 23 & 10 & 1 & 132 \\
\hline
\end{tabular}


Tabela 3. Modelos volumétricos utilizados no ajuste de equações para determinação do volume de madeira em pé no PDS Virola-jatobá em Anapu, Pará.

\begin{tabular}{ccc}
\hline Modelo & Relação matemática & Autores \\
\hline 1 & $\mathrm{v}=\beta_{0}+\beta_{1} \mathrm{~d}+\varepsilon_{\mathrm{i}}$ & Berkhant \\
2 & $\mathrm{v}=\beta_{0}+\beta_{1} \mathrm{~d}^{2}+\varepsilon_{\mathrm{i}}$ & Koperzky e Gehrhardt \\
3 & $\mathrm{v}=\beta_{0}+\beta_{1} \mathrm{~d}+\beta_{2} \mathrm{~d}^{2}+\varepsilon_{\mathrm{i}}$ & Hohenadl e Krenn \\
4 & $\operatorname{logv}=\beta_{0}+\beta_{1} \log \mathrm{d}+\beta_{2} 1 / \mathrm{d}+\varepsilon_{\mathrm{i}}$ & Brenac \\
5 & $\log \mathrm{v}=\beta_{0}+\beta_{1} \log \mathrm{d}+\varepsilon_{\mathrm{i}}$ & Husch \\
6 & $\mathrm{v}=\beta_{0}+\beta_{1} \mathrm{~d} \mathrm{~d}^{2} \mathrm{~h}+\varepsilon_{\mathrm{i}}$ & Spurr \\
7 & $\operatorname{logv}=\beta_{0}+\beta_{1} \log \mathrm{d}+\beta_{2} \operatorname{logh}+\varepsilon_{\mathrm{i}}$ & Schumacher e Hall \\
8 & $\mathrm{v}=\beta_{0}+\beta_{1} \mathrm{~d}^{2}+\beta_{2} \mathrm{~d}^{2} \mathrm{~h}+\beta_{3} \mathrm{~h}+\varepsilon_{\mathrm{i}}$ & Stoate \\
9 & $\operatorname{logv}=\beta_{0}+\beta_{1} \log \mathrm{d}^{2} \mathrm{~h}+\varepsilon_{\mathrm{i}}$ & Spurr \\
10 & $\mathrm{v}=\beta_{0}+\beta_{1} \mathrm{~d}^{2}+\beta_{2} \mathrm{~d}^{2} \mathrm{~h}+\beta_{3} \mathrm{dh}^{2}+\beta_{4} \mathrm{~h}^{2}+\varepsilon_{\mathrm{i}}$ & Naslund \\
\hline
\end{tabular}

$\mathrm{v}=$ volume estimado em $\mathrm{m}^{3} ; \mathrm{d}=$ diâmetro tomado a $1,30 \mathrm{~m}$ do solo em $\mathrm{m} ; \mathrm{h}=$ altura comercial do fuste em $\mathrm{m} ; \beta_{0}, \beta_{1}, \beta_{2}, \beta_{3}, \beta_{4}=$ coeficientes da regressão; $\varepsilon_{\mathrm{i}}=$ termo de erro aleatório.

Para a seleção da melhor equação volumétrica analisou-se os seguintes critérios estatísticos:

O coeficiente de determinação $\left(\mathrm{R}^{2}\right)$ expressa a quantidade da variação total que é explicada pela regressão (Machado et al., 2008), sendo calculado pela expressão:

\section{$\mathrm{R}^{2}=$ [1 - (SQresíduo /SQtotal) ]}

Em que $\mathrm{R}^{2}$ = coeficiente de determinação; $\mathrm{SQ}_{\text {residuo }}=$ soma de quadrados de resíduos da regressão; e $\mathrm{SQ}_{\text {total }}=$ soma de quadrados totais da regressão.

Os valores de $\mathrm{R}^{2}$ foram reajustados para que os modelos fossem comparáveis, tendo em vista os diferentes graus de liberdades entre os modelos testados. O coeficiente de determinação ajustado foi calculado através da expressão matemática:

\section{$R^{2} a j=1$ - [((n-1)/(n-p)).(SQresíduo/SQtotal) $]$}

Em que $\mathrm{R}_{\text {aj }}^{2}=$ coeficiente de determinação ajustado; $\mathrm{n}=$ número de dados observados; $p=$ número de coeficientes do modelo; $\mathrm{SQ}_{\text {residuo }}=$ soma de quadrados de resíduos da regressão; e $\mathrm{SQ}_{\text {total }}=$ soma de quadrados totais da regressão.

Foi calculado o valor de F, obtido por meio da análise de variância da regressão, que tem por finalidade indicar se existe diferença significativa no ajuste a determinado nível de probabilidade, por meio da comparação do valor de $\mathrm{F}$ calculado com o valor de $\mathrm{F}$ tabelado. Também foi determinado o erro padrão da estimativa, que indica a precisão do ajuste de um modelo matemático ao medir a dispersão média entre os valores observados e estimados ao longo da linha da regressão. O erro padrão da estimativa foi calculado através da expressão matemática:

\section{$\mathrm{Sxy}=\sqrt{\text { QMresíduo }}$}

Em que $\mathrm{S}_{\mathrm{yx}}=$ erro-padrão da estimativa; $\mathrm{QM}_{\mathrm{res}}=$ quadrado médio do resíduo, obtido na análise da variância.

Quando a variável dependente sofre algum tipo de transformação, é necessário que se faça o recálculo do $\mathrm{S}_{\mathrm{yx}}$, para que se possam comparar estatisticamente as equações que foram transformadas com as outras que não foram (Silva, 2007b). Esse recálculo é efetuado pela seguinte fórmula:

$\mathrm{Sxyr}=\sqrt{\sum_{\mathrm{i}=1}^{\mathrm{n}}(\mathrm{yi}-\hat{y})^{2} /(n-p)}$

Em que Sxyr = erro-padrão da estimativa recalculado; yi = valor real de cada observação; $\hat{y} \mathrm{i}=$ valor estimado de cada observação; $\mathrm{n}=$ número de dados; $\mathrm{p}=$ número de coeficientes de cada equação, incluindo b0.

O coeficiente de variação $(\mathrm{CV} \%)$ foi obtido pela relação:

$\mathrm{CV}(\%)=\frac{\mathrm{Sxyr}}{\hat{Y}} .100$

Onde Sxyr = erro-padrão da estimativa recalculado,

$\hat{Y}=$ média aritmética da variável dependente.

A análise da distribuição gráfica dos valores residuais é um critério importante, pois possibilita a verificação do ajuste da equação ao longo da amplitude dos dados, permitindo detectar se há ou não tendenciosidade na estimativa da variável dependente ao longo da linha de regressão, ou seja, se a dispersão dos pontos ao longo do 
eixo da variável independente indica de forma clara se o ajuste subestima ou superestima a variável dependente. Sendo determinante na avaliação da qualidade das estimativas, para tomada de decisão quanto à utilização ou não do modelo (Rufino et al., 2010).

Os resíduos de um modelo de regressão linear têm uma relação muito forte com a qualidade do ajuste feito, bem como a confiabilidade dos testes estatísticos sobre os parâmetros do modelo, ou seja, é imprescindível para verificação da qualidade dos ajustes de modelos (Charnet et al., 2008). Além disso, a análise de resíduos permite estudar a heterogeneidade de variâncias, a independência de erros e a presença de outliers (Resende, 2007).

Ademais, para as equações logarítmicas foi determinado o fator de correção de Meyer (FM), através da fórmula $\mathrm{FM}=\mathrm{e}^{\left(0,5 * \mathrm{Syx}^{2}\right)}$, em que: $\mathrm{e}=$ constante de Euler $(2,718281828 \ldots)$; e Syx $=$ o erro padrão de estimativa, como fins de minimizar a discrepância logarítmica que, em geral, subestima a variável resposta (Baskerville, 1971). A significância dos coeficientes dos modelos ajustados foi avaliada pelo teste $\mathrm{t}(p<0,01)$.

Após o ajuste e seleção dos melhores modelos de simples e dupla entrada foi utilizado e teste Quiquadrado $(\alpha=0,01 ; \mathrm{gl}=39)$ para validar os modelos selecionados, pela relação $x^{2}=\sum \frac{(y i-\hat{y} i)^{2}}{\hat{y} i}$ em uma base de dados composta de 40 indivíduos não utilizados para o ajuste dos modelos. As equações selecionadas foram validadas através de três comparações:

Volume real (Yi) e volume estimado pelo melhor modelo de simples entrada ( $\hat{\mathrm{Y}} \mathrm{i})$;

Volume real (Yi) e volume estimado pelo melhor modelo de dupla entrada $(\hat{Y} i)$;
Volume estimado (Yi) pelo melhor modelo de dupla entrada e volume estimado pelo melhor modelo de simples entrada (YYi).

\section{Resultados e discussão}

$\mathrm{O}$ volume real dos indivíduos utilizados para o ajuste dos modelos teve média e desvio padrão de 10,01 $\pm 5 ., 22$ $\mathrm{m}^{3} \mathrm{e}$ um total de $1.572,52 \mathrm{~m}^{3}$. A variável DAP teve média e desvio padrão de $0,89 \pm 0 ., 16 \mathrm{~m}$, e a variável h apresentou média e desvio padrão de $20,8 \pm 5 ., 93 \mathrm{~m}$. O fator de forma médio obtido foi de 0,75 variando entre 0,52 e 0,97.

Os modelos de Berkhant, Koperzky e Gehrhardt e Hohenadl e Krenn apresentaram alto CV\%, com, aproximadamente, $34 \%, 32 \%$ e $30 \%$ dos dados de volume real, respectivamente. Os modelos de Berkhant, Koperzky e Gehrhardt apresentaram uma tendência sistemática negativa na distribuição de seus resíduos, superestimando o volume nas primeiras classes de diâmetro, já os modelos de Hohenadl e Krenn apresentaram uma distribuição de resíduos homogênea aleatória na média zero (Figura 2; Tabela 4).

Os modelos de Brenac e Husch apresentaram valores dos parâmetros da regressão satisfatórios, semelhantes entre si e uma distribuição de resíduos aleatória e homogênea em torno da média zero, tornando os dois modelos ajustados muito precisos, semelhante ao encontrado por Rolim et al. (2006) na Floresta Nacional do Tapirapé-Aquir na Serra dos Carajás, Pará. Brenac apresenta $\beta_{0}$ e $\beta_{1}$ não significativos, levando a escolha do modelo proposto por Husch como a equação de simples entrada ajustada com maior precisão (Figura 1; Tabela 4).

Tabela 4. Parâmetros e coeficientes dos modelos volumétricos ajustados para o PDS Virola-jatobá em Anapu, Pará.

\begin{tabular}{ccccccccccc}
\hline Modelo & $\boldsymbol{\beta}_{\mathbf{0}}$ & $\boldsymbol{\beta}_{\mathbf{1}}$ & $\boldsymbol{\beta}_{\mathbf{2}}$ & $\boldsymbol{\beta}_{\mathbf{3}}$ & $\boldsymbol{\beta}_{\mathbf{4}}$ & $\mathbf{R}^{\mathbf{2}} \mathbf{a j}$ & $\mathbf{S y x r}$ & $\mathbf{C V} \%$ & $\mathbf{D M P} \%$ & $\mathbf{F M}$ \\
\hline 1 & $-14,12$ & 21,3085 & & & & 0,79 & 1,76 & 34,76 & 8,51 & \\
2 & $-5,0157$ & 12,0418 & & & & 0,82 & 1,63 & 32,20 & 5,31 & \\
3 & 12,3258 & 39,6589 & 33,9794 & & & 0,84 & 1,53 & 30,33 & $-4,83$ & \\
\hline 4 & $0,8807^{*}$ & $4,2053^{*}$ & 0,0828 & & & 0,93 & 0,09 & 17,54 & 4,81 & 1.0001 \\
\hline 5 & 0,7974 & 4,4294 & & & & 0,93 & 0,09 & 17,48 & 5,11 & 1.0002 \\
\hline 6 & $-2,0543$ & 0,3862 & & & & 0,84 & 1,54 & 30,47 & $-4,19$ & \\
7 & $-0,0295^{*}$ & 4,1170 & 0,6168 & & & 0,96 & 0,07 & 12,91 & 1,94 & 1.0000 \\
\hline 8 & $1,2637^{*}$ & $0,4207^{*}$ & 0,4972 & 0,2679 & & 0,91 & 1,15 & 22,79 & 3,50 & \\
9 & $-1,2692$ & 1,5165 & & & & 0,87 & 0,13 & 23,38 & 0,12 & 1.0000 \\
10 & $1,6125^{*}$ & $0,5888^{*}$ & 0,4840 & $0,0002^{*}$ & $0,0058^{*}$ & 0,90 & 1,17 & 23,23 & 4,52 & \\
\hline
\end{tabular}

* Coeficientes não significativos pelo teste $\mathrm{t}(p<0,01)$. 
Dentre os modelos de dupla entrada (Figura 1; Tabela 4), o de Spurr apresentou baixo $R^{2}$ aj, ou seja, somente $84 \%$ da variável volume foi explicado pelo modelo de regressão linear ajustado e também apresentou valores altos nos parâmetros Syx e CV(\%). O modelo de Spurr obteve uma heterogeneidade da variância nos resíduos. Os modelos de Stoate, logarítmico de Spurr e de Naslund apresentaram alto $\mathrm{CV}(\%)$ e heterocedasticidade na distribuição de resíduos, sendo que o de Naslund teve muitos coeficientes não significativos, o que pode ser explicado pela presença da multicolinearidade (Valente et al., 2011).

O modelo de dupla entrada proposto por Schumacher e Hall apresentou maior $\mathrm{R}^{2}$ aj, menores valores de Syx e $\mathrm{CV}(\%)$ e distribuição não tendenciosa de resíduos tornando-se um modelo muito preciso (Figura 1; Tabela 4), similar ao encontrado por Silva \& Araújo et al., (1984) na Floresta Nacional de Tapajós, Pará; Rolim et al., (2006) na Floresta Nacional do Tapirapé-Aquir na Serra dos Carajás, Pará e por Thaines et al., (2010) em uma floresta no município de Lábrea no Amazonas. Esse modelo apresentou $\beta_{0}$ não significativo $(p=0,7108)$, ou seja, o coeficiente que representa o valor da variável dependente quando as variáveis independentes forem iguais a zero. Por não ser significativo, não influenciou nos volumes estimados pelo modelo, tendo sido eliminado da equação. Esse modelo foi confirmado como o melhor dentre os de dupla entrada.

O modelo proposto por Schumacher e Hall mostrou-se mais preciso que o de Husch apresentando parâmetros melhores e erros mais próximos de zero, mostrando que a introdução da variável altura (h) melhorou de forma significativa a precisão da equação de volume ajustada. Ambos subestimaram o volume, resultado este que se assemelhou com os de Rolim et al., (2006) na Floresta Nacional do Tapirapé-Aquir na Serra dos Carajás, Pará e em uma floresta densa no município de Lábrea no Amazonas o que é preferível a superestimar, quando se trata do planejamento do manejo florestal comunitário (Thaines et al., 2010).

Barros \& Silva Júnior, (2009) em uma floresta localizada no município de Anapu, Pará, no mesmo munícipio do atual estudo, verificaram que as equações que melhor se ajustaram foram os modelos de Brenac como de simples entrada e o modelo de Spurr como de dupla entrada, resultados estes muito diferentes aos encontrados pelo atual estudo, porém vale ressaltar que Barros \& Silva Júnior (2009) não analisaram a significância dos coeficientes e a distribuição dos resíduos, o que pode ter influenciado na escolha final da melhor equação.
Não houve diferença significativa entre o volume estimado pelo modelo de Husch e o volume real $\left(\mathrm{X}_{\text {calc }}{ }^{2}=\right.$ $44,93 ; p=0,23)$; entre o volume estimado pelo modelo de Schumacher e Hall e o volume real $\left(\mathrm{X}_{\text {calc }}^{2}=33,42 ; p\right.$ $=0,72)$ e entre os volumes estimados pelos modelos de Husch e Schumacher e Hall $\left(\mathrm{X}_{\text {calc }}{ }=10,67 ; p=0,99\right)$, provando que as duas equações estão aptas para estimar o volume de madeira em pé no PDS Virola-jatobá.

O modelo proposto por Schumacher e Hall pode ser utilizado para estimar o volume de madeira em pé no PDS Virola-jatobá, mas utiliza a variável altura comercial, o que pode onerar a etapa de inventário florestal, aumentar o tempo de trabalho em campo e incluir erros sistemáticos intrínsecos a mensuração dessa variável. Neste caso, o modelo de simples entrada proposto por Husch se torna mais prático para execução desta etapa pelos comunitários do PDS Virola-Jatobá ao mesmo tendo um ajuste inferior a o modelo de dupla entrada proposto por Schumacher e Hall. O menor ajuste dos modelos de simples entrada é esperado, uma vez que esses modelos assumem que árvores do mesmo diâmetro tem a mesma altura, o que não é verdadeiro para florestas tropicais (Rolim et al., 2006).

O modelo do volume cilíndrico utilizando o fator de forma 0,7 subestima os volumes das árvores do PDS Virola-Jatobá, que apresentou fator de forma 0,74 , valores estes muito próximos ao encontrado por Rolim et al. (2006) e Thaines et al., (2010). Segundo Thaines et al., (2010), os valores de fator de forma não se relacionam com os diâmetros, podendo assumir qualquer valor em qualquer classe dificultando a sua utilização como aferidor volumétrico definitivo, tornando imprescindível a utilização de equações volumétricas específicas para cada localidade.

\section{Conclusões}

O modelo de Husch é apropriado para estimar o volume de madeira em pé no empreendimento florestal do Projeto de Desenvolvimento Sustentável VirolaJatobá, através da equação $\log v=0,7974+4,4294 \log$. Além de ter precisão aceitável, esse modelo pode gerar menores custos, menor tempo e menor erro na etapa de inventário florestal para os comunitários, pois utiliza apenas o DAP como variável dendrométrica, tornandose mais prática para comunidade do PDS Virolajatobá. Vale ressaltar também que o ajuste de equações volumétricas pontuais é importante para se estimar o volume de madeira com maior precisão em locais de manejo comunitário. 
Berkhant

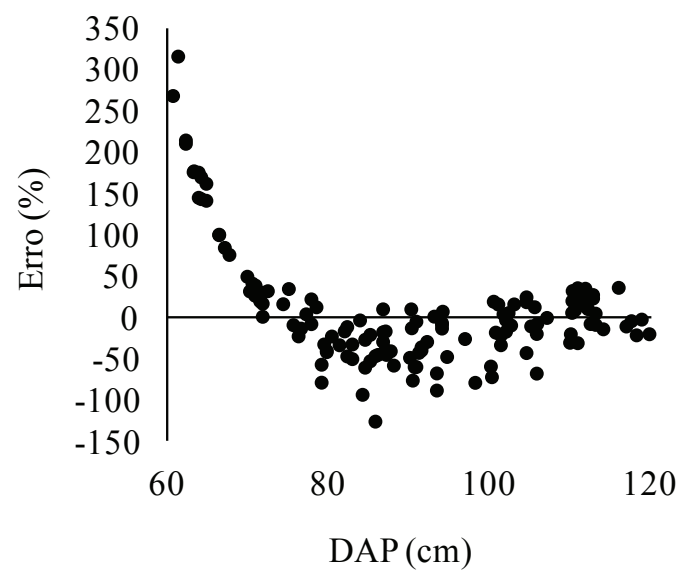

Hohenadl e Krenn

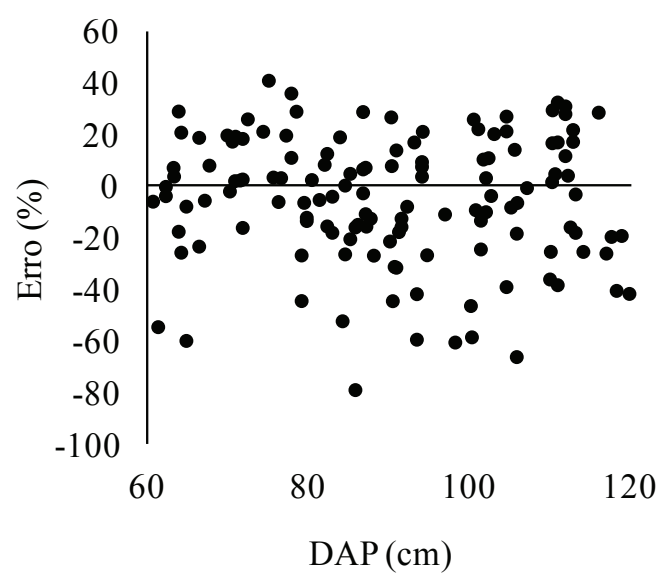

Husch

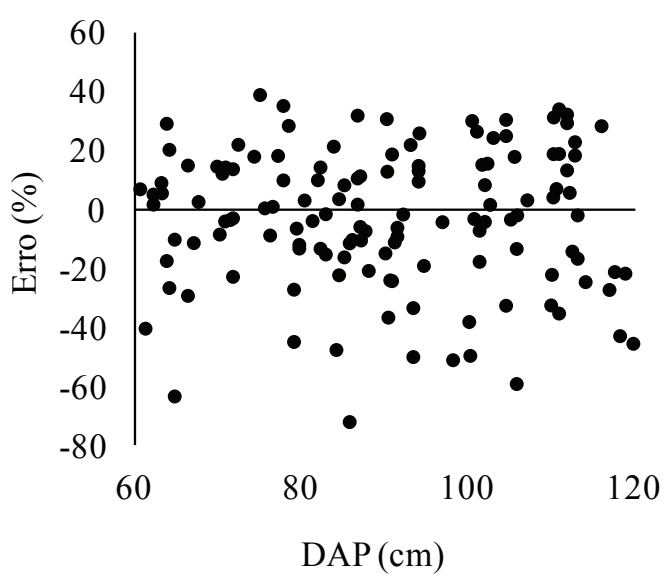

Koperzky e Gehrardt

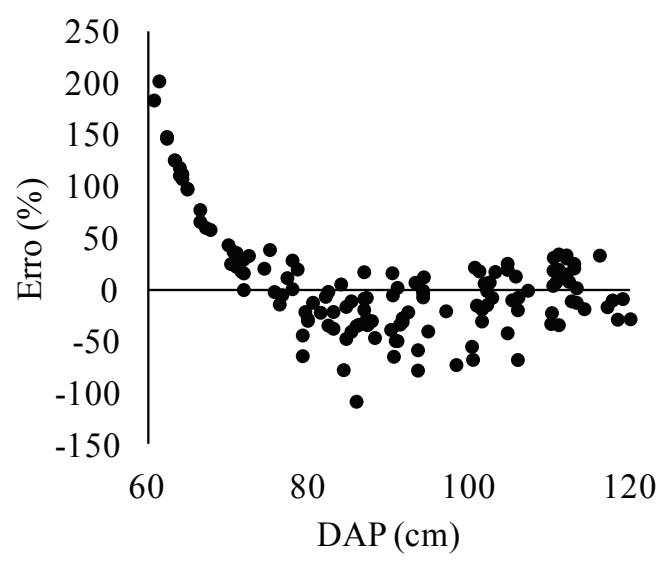

Brenac

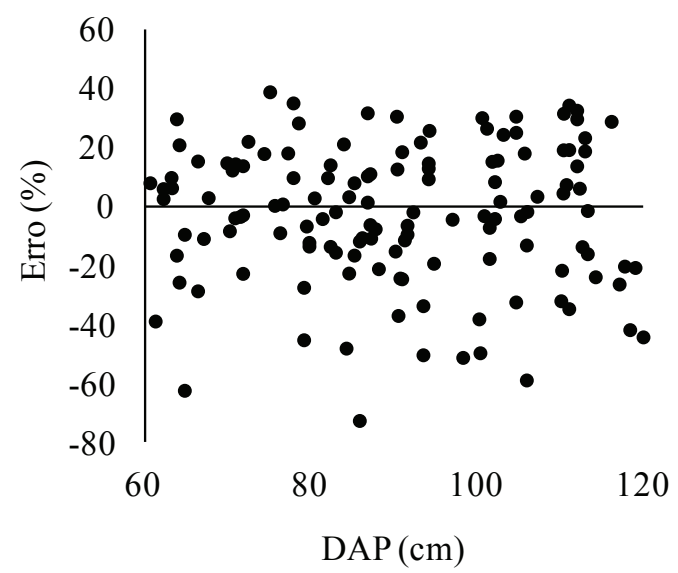

Spurr

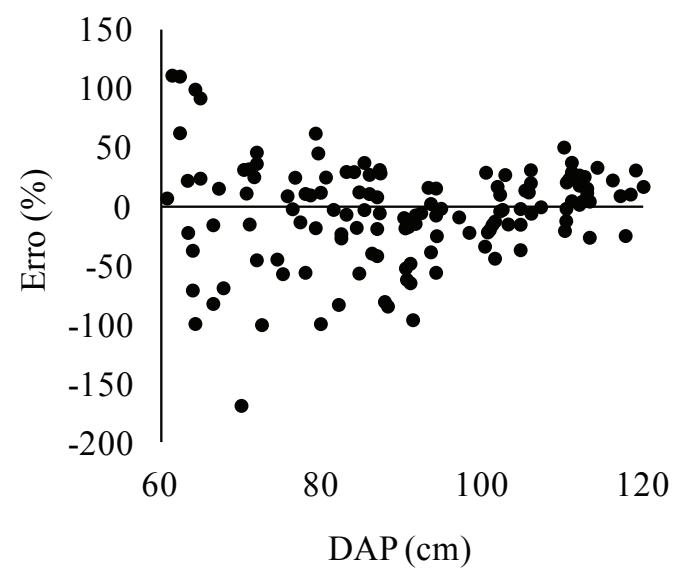

Figura 1. Distribuição de resíduos dos modelos utilizados para o ajuste de equações para determinação do volume de madeira em pé no PDS Virola-Jatobá em Anapu, Pará. 

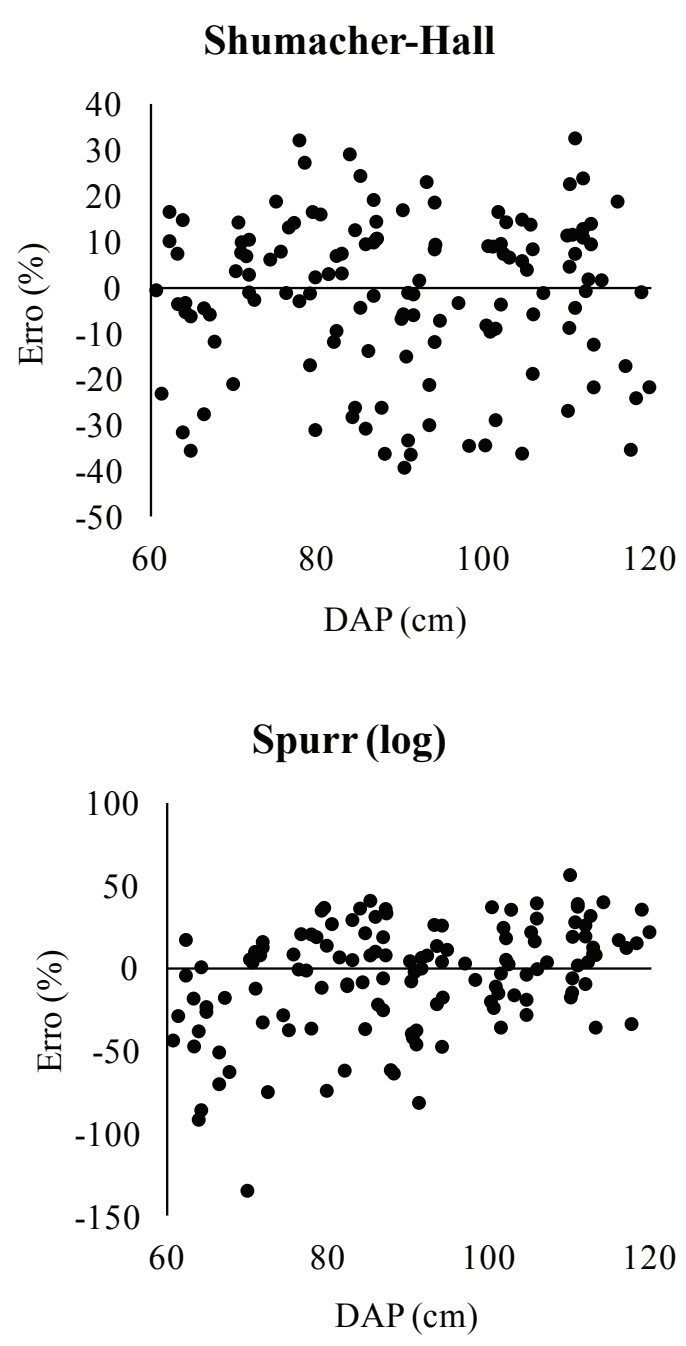

Figura 1. Continuação.

\section{Referências}

ANGIOSPERM PHYLOGENY GROUP. An update of the angiosperm phylogeny group classification for the orders and families of flowering plants: APG II. Botanical Journal of the Linnean Society, London, v. 141, n. 4, p. 399-436, 2003.

BARROS, P. L. C.; SILVA JÚNIOR, A. T. Equação de volume para árvores de uma floresta tropical densa no município de Anapu, oeste do estado do Pará, Amazônia oriental. Revista de Ciências Agrárias, v. 51, p.115-126, 2009.

BASKERVILLE, G. L. Use of logarithmic regression in the estimation of plant biomass. Canadian Jornal Forest Research, v. 2, p. $49-53,1972$.

BRASIL. Resolução CONAMA no 406 de 02 de fevereiro de 2009. Estabelece parâmetros técnicos a serem adotados na elaboração, apresentação, avaliação técnica e execução de Plano de Manejo Florestal Sustentável- PMFS com fins madeireiros, para florestas nativas e suas formas de sucessão no bioma Amazônia. Diário Oficial [da] República Federativa do Brasil, Brasília, DF, n. 26, 06 de fev. 2009.
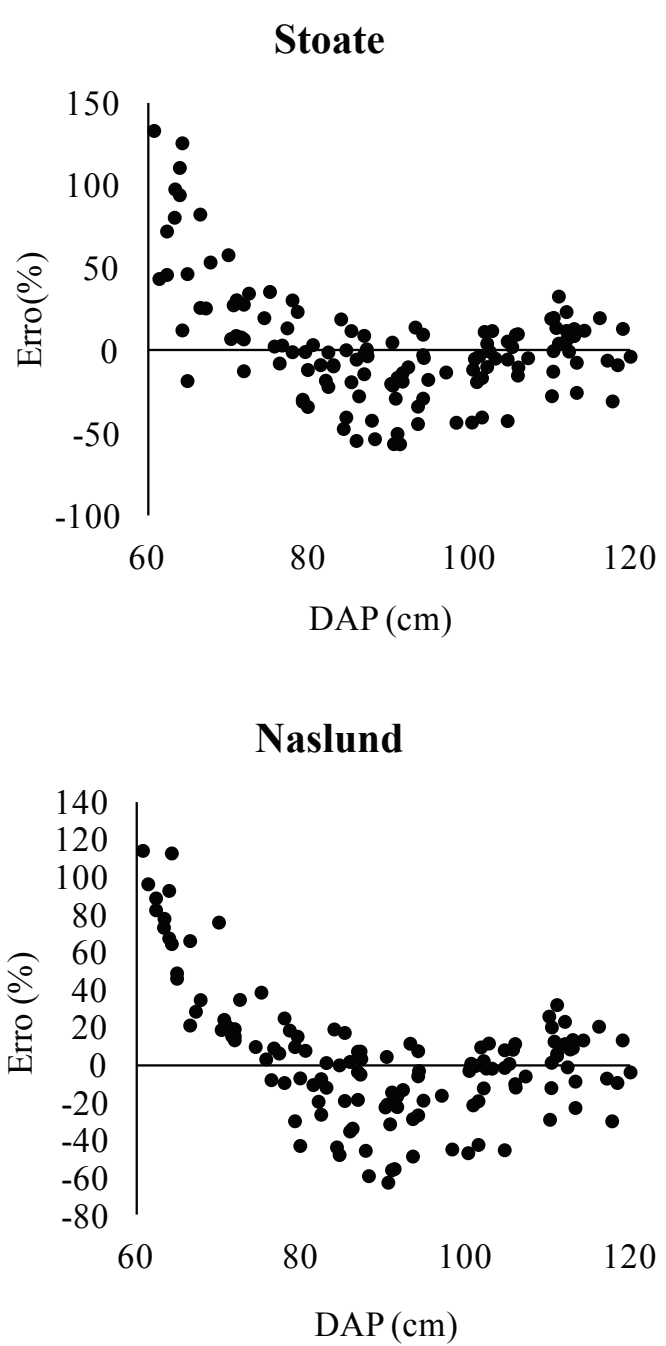

CHARNET, R.; FREIRE, C. A. L.; CHARNET, E. M. R.; BONVINO, $H$. Análise de modelos de regressão linear: com aplicações. 2. ed. Campinas: Ed. da Unicamp, 2008.

COLPINI, C.; TRAVAGIN, D. P.; SOARES, T. S.; SILVA, V. S. M. Determinação do volume, do fator de forma e da porcentagem de casca de árvores individuais em uma Floresta Ombrófila Aberta na região noroeste de Mato Grosso. Acta Amazonica, Manaus, v. 39, n. 1, p. 97-104, 2009

FERNANDES, N. P.; JARDIM, F. C. S.; HIGUCHI, N. Tabelas de volume para a floresta de terra firme da Estação Experimental de Silvicultura Tropical. Acta Amazonica, Manaus, n. 13, v. 3-4, p. 537-545, 1983.

FIGUEIREDO FILHO, A. Estudos de modelos matemáticos para estimar o volume por unidade de área em uma floresta tropical úmida na Amazônia brasileira. 1983. Dissertação (Mestrado em Ciências) - Universidade Federal do Paraná, Curitiba.

INCRA (Brasília, DF). Instrução normativa ${ }^{\circ} 65$ de 27 de Dezembro de 2010. Estabelece critérios e parâmetros para as atividades de manejo florestal sustentável em projetos de assentamento. Diário Oficial [da] República Federativa do Brasil, Brasília, DF, n. 251, 31 dez. 2010. 
LOETSCH, F.; ZÖHRER, F.; HALLER, K. E. Forest inventory. Reinbek: Federal Research Organization for Forestry and Forest Products, Forest Inventory Section, 1973. 469 p. v. 2.

MACHADO, S. A.; NASCIMENTO, R. G. M.; AUGUSTYNCZIK, A. L. D.; SILVA, L. C. R.; FIGURA, M.A.; PEREIRA, E.M.; TÉO, S.J. Comportamento da relação hipsométrica de Araucaria angustifólia no capão da Engenharia Florestal da UFPR. Pesquisa Florestal Brasileira, Colombo, n. 56. p. 5-16, 2008.

MACHADO, S. do A.; FIGUEIREDO FILHO, A. Dendrometria. Guarapuava: UNICENTRO, 2009. 316 p.

PARÁ. Projeto de Desenvolvimento Sustentável dos Assentamentos III e IV (Virola Jatobá) no município de Anapu-PA. INCRA. 2004.

RESENDE, M. D. V. Matemática e estatística na análise de experimentos e no melhoramento genético. Colombo: Embrapa Florestas, 2007.

ROLIM, S. G.; COUTO, H. T. Z.; JESUS, R. M.; FRANÇA, J. T. Modelos volumétricos para a Floresta Nacional do Tapirapé-Aquirí, Serra dos Carajás (PA). Acta Amazonica, Manaus, v. 36, v. 1, p. 107-114, 2006

RUFINO, R. F.; MIGUEL, E. P.; SANTOS, G. A.; SANTOS, T. E. B.; SOUZA, F. Ajuste de modelos hipsométricos para um povoamento de eucaliptos conduzido sobre o sistema de rebrota. EnciclopédiaBiosfera, Goiânia, v. 6, n. 10, p. 1-10, 2010.

SANTOS, A. T. dos; MATTOS, P. P. de; BRAZ, E. M.; ROSOT, N. C. Equação de volume e relação hipsométrica em plantio de Ocotea porosa. Pesquisa Florestal Brasileira, Colombo, v. 32, n. 69, p. 13-21, 2012. DOI: 10.4336/2012.pfb.32.69.13

SILVA, J. L. R da. Modelos volumétricos, fatores de forma e equação de afilamento para floresta de terra firme da região do rio Aru, município de Portel - Pará. 2007a. 20 f. Dissertação (Mestrado em Ciências Florestais) - Universidade Federal Rural da Amazônia, Belém.
SILVA, R. P. Alometria, estoque e dinâmica da biomassa de florestas primárias e secundárias na região de Manaus(AM). 2007b. 38 f. (Tese de Doutorado em Biologia Tropical e Recursos Naturais) - Instituto Nacional de Pesquisas da Amazônia/ Universidade Federal do Amazonas, Manaus.

SILVA, J. N. M.; ARAÚJO, S. M. Equação de volume para árvores de pequeno diâmetro, na Floresta Nacional do Tapajós. Boletim de Pesquisa Florestal, Colombo, n. 8/9, p. 16-25, 1984.

SILVA, J. N. M.; CARVAlHO, J. O. P de; LOPES, J. do C. A.; CARVALHO, M. S. P. de; Equações de volume para a Floresta Nacional do Tapajós. Boletim de Pesquisa Florestal, Colombo, n. 8/9, p. 50-63, 1984.

SILVA, J. N. M.; CARVALHO, M. S. P. Equações de volume para uma floresta secundária no Planalto do Tapajós-Belterra, Pa. Boletim de Pesquisa Florestal, Colombo, n. 8/9, p. 1-15, 1984.

SILVA JÚNIOR, A. T. Equação de volume e fator de forma para árvores de Schizolobium parahyba var. Amazonicum (Huber ex Ducke) Barneby (Paricá) no município de Aurora do Pará. 2009. 09 f. Dissertação (Mestrado em Ciências Florestais). Universidade Federal Rural da Amazônia.

THAINES, F.; BRAZ, E. M.; MATTOS, P. P.; THAINES, A. A. R. Equações para estimativa do volume de madeira para a região da bacia do Rio Ituxi, Lábrea, AM. Pesquisa Florestal Brasileira, Colombo, v. 30, n. 64, p. 283-289, 2010.

THOMAS, C.; ANDRADE, C. M.; SCHNEIDER P. R.; FINGER, C. A. Comparação de equações volumétricas ajustadas com dados de cubagem e análise de tronco. Ciência Florestal, Santa Maria, RS, v. 16, n. 3. p. $319-327,2006$

VALENTE, M. D. R.; QUEIROZ, V. T de; PINHEIRO, J. G.; MONTEIRO, L. A. da S.; Modelo de predição para o volume total de Quaruba (Vochysia inundata Ducke) via análise de fatores e regressão. Revista Árvore, Viçosa, MG, v. 35, n. 2, p. 307-317, 2011. 
